\title{
OPERATION OF THE CDF SILICON VERTEX DETECTOR WITH COLLIDING BEAMS AT FERMILAB
}

F. Bedeschi, V. Bolognesi, S. Dell'Agnello, S. Galeotti, G. Grieco, M Mariotti, A. Menzione, G. Punzi, F. Raffaelli, L. Ristori, F. Tartarelli, N. Turini,

H. Wenzel, F. Zetti

INFN, University and Scuola Normale Superiore of Pisa, Pisa, Italy, I-56100

M. W. Bailey, A. F. Garfinkel, M. C. Kruse, N. M. Shaw

Purdue University, West Lafayette, Indiana, 47907

W. C. Carithers, R. Ely, C. Haber, S. Holland, S. Kleinfelder,

T. Merrick, O. Schneider, W. Wester, M. Wong, W. Yao

Lawrence Berkeley Laboratory, Berkeley, California, 94720

H. Carter, B. Flaugher, C. Nelson, S. Segler, T. Shaw,

S. Tkaczyk, K. Turner, T. R. Wesson

Fermi National Accelerator Laboratory, Batavia, Illinois, 60510

B. Barnett, C. Boswell, J. Skarha, F. D. Snider, A. Spies,

J. Tseng, S. Vejcik

The John Hopkins University, Baltimore, Maryland, 21218

D. Amidei, P. F. Derwent, T. Y. Song, A. Dunn

University of Michigan, Ann Arbor, Michigan, 48109

M. Gold, J. Matthews, N. Bacchetta

University of New Mexico, Albuquerque, New Mexico, 87131

P. Azzi, D. Bisello, G. Busetto, A. Castro, M. Loreti, L. Pescara

University of Padova, Padova, Italy, I-35100

P. Tipton, G. Watts

University of Rochester, Rochester, New York, 14627

\begin{abstract}
In this paper we briefly describe the main features of the CDF Silicon Vertex Detector (SVX) and discuss its performance during actual colliding beam operation at the Fermilab Tevatron. Details on $\mathrm{S} / \mathrm{N}$ ratio, alignment, resolution and efficiency are given.
\end{abstract}

\section{INTRODUCTION}

The Collider Detector at Fermilab (CDF) is a multipurpose detector designed and built to study $p \bar{p}$ interactions at $\sqrt{s}=2 \mathrm{TeV}^{1}$. Several upgrades have been added to the basic detector for the data taking run which has recently started; among these is a silicon mi- crostrip detector (SVX) to provide high resolution tracking in the interaction region. This detector will allow us to resolve secondary vertices from the decay of heavy-flavoured particles, with lifetimes in the range of $10^{-13}-10^{-12}$ seconds. We expect the SVX to give a fundamental contribution to the measurement of 
the properties of the particles containing the $b$ quark and to the identification of events containing the top quark, if its production rates are sufficiently high to allow detection.

Several technical papers have been presented in the past describing the $\mathrm{SVX}^{2,4,5,7}$, its front-end electronics ${ }^{10,11}$, the details of the construction ${ }^{6,8}$, the performance of prototypes during beam tests ${ }^{3,9}$, and the data acquisition system $^{12,13}$; in the following we will give only a brief description of the detector and then concentrate on how the full detector performs during actual data taking conditions at CDF, based on the information extracted from the first few months of data collection.

\section{THE SVX DETECTOR}

The SVX is installed surrounding the CDF interaction region inside the vertex time projection chamber (VTX) and the central tracking chamber (CTC). One of its most distinctive features is its length: over $50 \mathrm{~cm}$ divided in two longitudinal sections (barrels) which sit symmetrically about the average interaction point. This length is driven by the need for a reasonably good acceptance in spite of the rather long size (Gaussian distributed with $\sigma=35 \mathrm{~cm}$ ) of the Tevatron luminous region . Each barrel (Figure 1) is divided into $12 \mathrm{sec}$ tions (wedges) which subtend $30^{\circ}$ in azimuth. Each wedge consists of 4 layers of silicon detector modules (ladders) with silicon microstrips running parallel to the beam axis. To cope with the rather long length of the ladders without the number of readout channels getting out of control, three $8.5 \mathrm{~cm}$ long microstrip detectors are assembled on each ladder, their strips wirebonded between adjacent detectors, with a single ceramic readout hybrid circuit board (ear card) at one end. This configuration causes a pretty high capacitive load on the inputs to the front end electronics $(\sim 30 p F)$, still, as we shall see, a quite reasonable noise

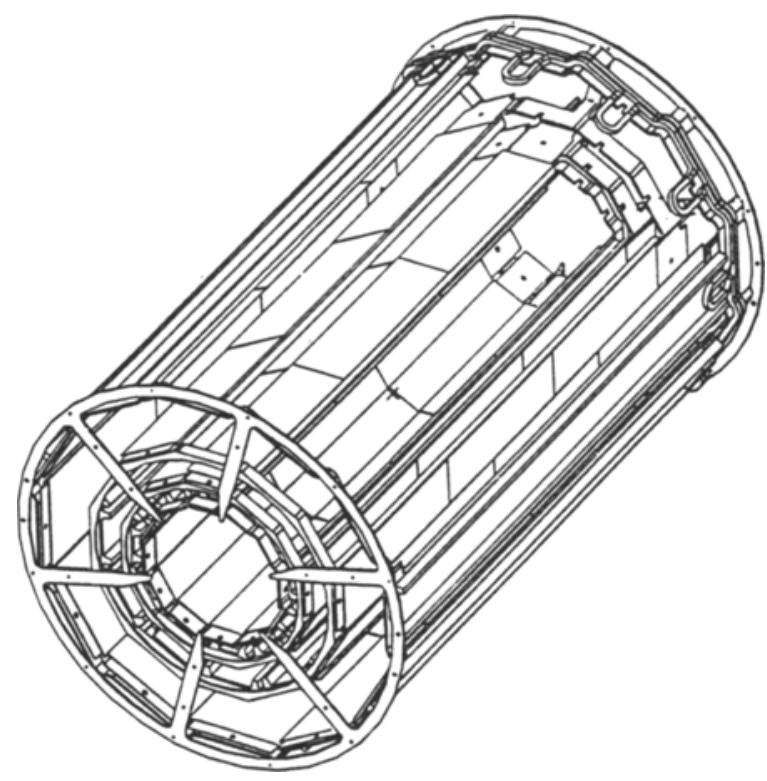

Figure 1. Schematic view of one SVX barrel, showing the internal geometry.

level can be achieved.

The main geometrical parameters of the SVX are the following: inner radius $2.99 \mathrm{~cm}$, outer radius $7.85 \mathrm{~cm}$, strip pitch $60 \mu \mathrm{m}$ for the the inner 3 layers and $55 \mu \mathrm{m}$ for the outer one. A total of 288 single-sided microstrip detectors are assembled into the system for a total of 46080 readout channels.

An effective chilled-water cooling ${ }^{6}$ system keeps the temperature of the detector in the $20-25^{\circ} \mathrm{C}$ range with a good safety margin.

\section{DETECTOR CHARACTERIZATION}

Special calibration runs are taken periodically between $p \bar{p}$ data-taking to monitor detector performance and calculate constants to be used later on in the data analysis. In particular we monitor the pedestal distribution, the gain and the sparsification thresholds for each channel. The results, after about 2 months of 
running, appear remarkably stable with time. The average noise measured is $12 \mathrm{ADC}$ counts, equivalent to about 2000 electrons. This noise is very uniform, within a few $A D C$ counts, across the whole detector. A uniformity well within $5 \%$ is also observed for the gains. Somewhat less satisfactory is the threshold uniformity for a few wedges. Since we have only two threshold setting lines per wedge, one for layer 1 and one for layers 2, 3 and 4, this results in a higher number of channels being read out in a given wedge, if we want to preserve a good efficiency for all of the channels. Currently the typical fraction of channels being read out per wedge is less than $5 \%$, and about $15 \%$ for a couple of wedges. Still this is of no consequence to the quality of the data.

The number of channels in the detector which do not respond properly (bad channels) is also monitored constantly. The fraction of bad channels is about $1 \%$, these channels being mostly either disconnected due to high strip currents, or low gain or high noise. This fraction has been rather stable over a period of time of several months. Alarge number of the bad channels found had already been detected during initial ladder testing, before the detector was assembled.

\section{RESULTS WITH TRACKS}

At present tracks in the SVX are found only as extrapolations of CTC tracks. The algorithm used associates one SVX point at a time to an existing CTC track. At each iteration a new fit of the track is made, multiple Coulomb scattering contributions are included and a new (narrower) search road is estimated to search for hits in the next layer. The algorithm works its way from the outer to the inner layer. In the end only tracks with 3 or more SVX hits are saved.

We have used the charge associated with hits connected to 4-hit tracks to study the en-

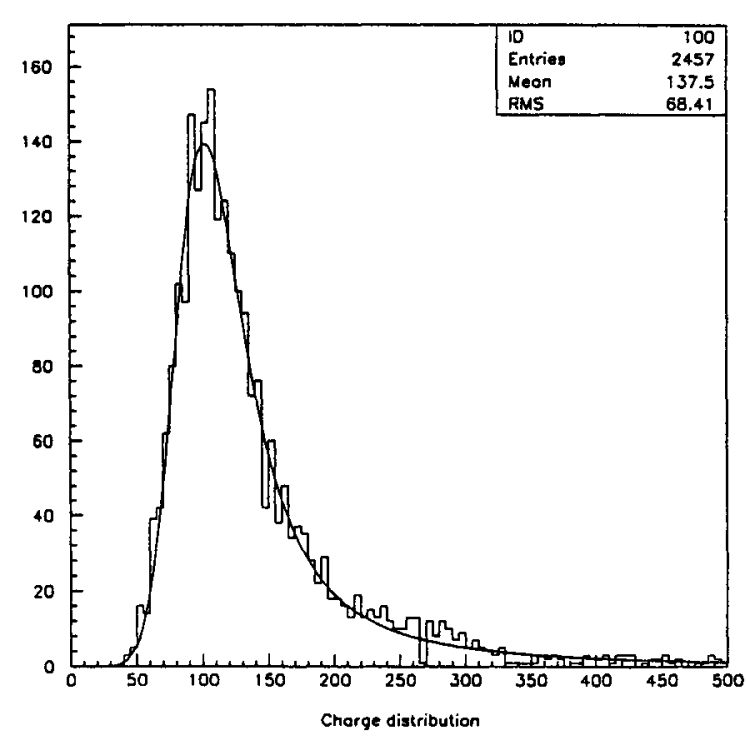

Figure 2. Charge distribution on layer 1 normalized to normal incidence (ADC counts).

ergy release of charged particles in the individual SVX layers. This selection allows very good noise rejection and the normalization of the collected charge to normal incidence angle. A typical distribution, quite consistent with a Landau shape, is shown in figure 2. The most probable value of this distribution is about $110 \mathrm{ADC}$ counts, corresponding to a worst case $\mathrm{S} / \mathrm{N}$ of 9 to 1 .

The efficiencies of the individual layers have been estimated by selecting all tracks with hits in the same wedge and then calculating the ratio between the number of tracks which miss a given layer, $N 1$, and the sum of $N 1$ and the number of all 4-hit tracks. This procedure is quite safe for the two intermediate layers, since the presence of hits on the first and last layer protects us from acceptance problems. The inefficiency obtained this way is about $3 \%$ for layers 2 and 3 of barrel 0 and 
$4 \%$ for layers 2 and 3 of barrel 1. These inefficiencies are higher than those measured in the testbeam $^{3,9}$ and possibly connected with the sparsification threshold settings. Work however is still in progress on the optimization of these settings and these numbers may still improve. In any case, due to the intrinsic redundancy of a 4-layer system, we still expect an overall tracking efficiency of $\sim 99 \%$ with the detector as it is, if we exclude acceptance effects.

\section{DETECTOR ALIGNMENT}

Though great care was taken in the construction of the $\mathrm{SVX}^{6}$ to keep the maximum construction error to a minimum, the detector needs careful alignment if the design spatial resolution of $12 \mu \mathrm{m}$ is to be achieved. Two types of alignment have been performed so far: first the alignment of each individual barrel to the CTC (global alignment) and then the internal alignment of the ladders within each barrel. We took great care in developing an alignment technique which concentrates the effect of the CTC systematics only in the global alignment step, where a lower accuracy is needed.

The global alignment method relies on the assumptions that both SVX barrels and the CTC must see the beam line in the same spot during a given run, and that each barrel can be treated as a rigid body. 5 alignment parameters must be determined for each barrel since the SVX does not measure $\mathrm{z}$, the longitudinal coordinate. These parameters are 3 rotations and $\mathrm{x}$ and $\mathrm{y}$ displacements. The beamline position as a function of $\mathrm{z}$ is calculated first using CTC tracks and then using tracks crossing only one barrel at a time. The difference in the beamline positions determines the transverse displacements and 2 rotation angles between the SVX and the CTC (the latter being used as a reference). The last parameter remain-

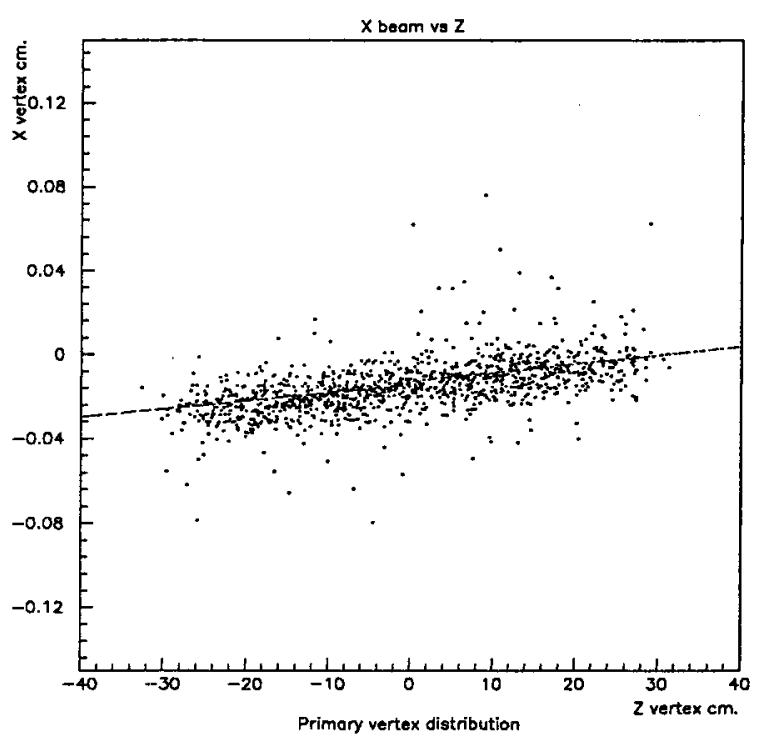

Figure 3. Beam line profile from the two SVX barrels.

ing is the relative rotation around $z$, which is determined by optimizing the match between the CTC track extrapolation to the SVX and the location of the SVX hits. As a check of the quality of this procedure an independent fit of the beam line is performed for the two barrels using tracks fully contained in either barrel, after applying the alignment corrections. The results so far differ by $\sim 15 \mu \mathrm{m}$ between the two barrels. Figure 3 shows the distribution of the $x$ versus $z$ of the primary vertex as obtained from this test and the fits performed for each barrel.

A great care was put, during construction, in building the SVX to a high level of mechanical accuracy. In addition, the misalignments have been measured with optical methods during assembly and these constants are already used for the track reconstruction. This gives us a very good starting point for the determination of the internal alignments using the 
SVX Residuals before and after internal alignment

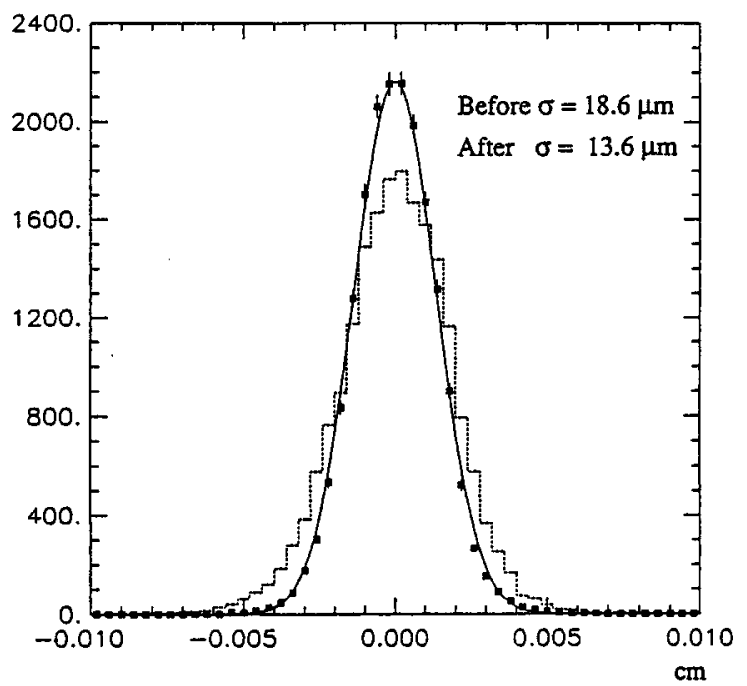

Figure 4. SVX residuals before and after internal alignment corrections.

data and allows some useful simplifications of the process. Some preliminary work has been done on internal alignment using tracks, and, though incomplete, it has already produced some good results. The method used is to first identify the degrees of freedom which most affect the resolution, to reduce the number of parameters to determine to a minimum. For each ladder we just fit the following parameters: a translation parallel to the detector plane and perpendicular to the strip direction, and a rotation around the axis perpendicular to the detector plane. To cope with the intrinsic degeneracy of the problem, we hold the outermost layer fixed and impose the constraint of the average beam line. We then fit the misalignment parameters on a wedgeby-wedge basis. Wedge-to-wedge corrections still remain to be studied, due to the large statistics needed, since one has to select tracks

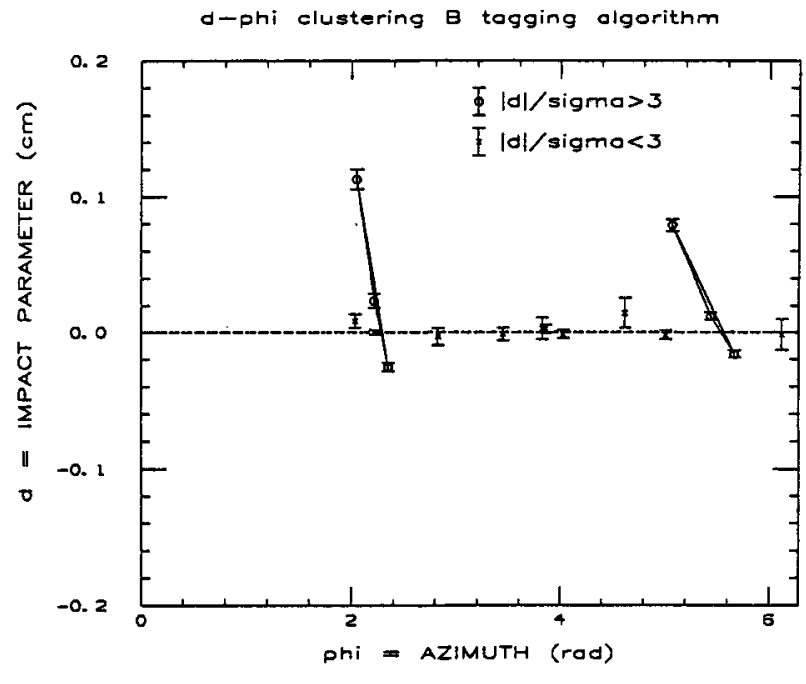

Figure 5. Typical event showing secondary vertex activity.

crossing wedge boundaries. Also the procedure needs to be iterated to stabilize the correlations, however small, between the global and internal alignment procedures. In figure 4 we show how the track residuals in the SVX improve after the internal alignment constants are applied. The width of the residual distribution improves from $18.6 \mu \mathrm{m}$ to $13.6 \mu \mathrm{m}$. The width of this residual distribution corresponds to a detector spatial resolution of 17 $\mu \mathrm{m}$, as verified with a simple toy Montecarlo.

\section{CONCLUSIONS}

In conclusion the SVX is already performing very close to the design expectations, and improvements are still possible. We have searched some fraction of our latest jet trigger data for indications of secondary vertex activity, and found, through a selection based only on the SVX, several candidates. Figure 5 
shows an example of two back-to-back vertices, each of multiplicity 3. In this figure we plot, for each track in the event, the impact parameter with respect to the reconstructed primary vertex versus the angle of the track with respect to the $\mathrm{x}$ axis, $\phi_{0}$. Tracks originating from a common vertex appear aligned in this plot, their intersection with the $\phi_{0}$ axis being the decaying particle direction. Tracks from the primary vertex lie on the $\phi_{0}$ axis. While much work and statistics are still needed to demonstrate that we are indeed observing heavy flavoured particle decays, the quality of events like this makes us believe that the SVX will play a very important role in the study of heavy flavour physics from the data collected during this period at CDF.

\section{REFERENCES}

1. F. Abe, et al., "The CDF Detector: An Overview", Nucl. Instrum. Methods A271, p. 387 (1988).

2. F. Bedeschi, et al., "A Silicon Vertex Detector for CDF", IEEE Trans. on Nucl. Sci. NS-33, p. 140 (1985).

3. F. Bedeschi, et al., "CDF Silicon Detector Prototype Test Beam Results", IEEE Trans. on Nucl. Sci. NS-36, p. 35 (1989).

4. C. Haber, "The CDF Silicon Microstrip Vertex Detector", in Proceedings of the $7^{\text {th }}$ Topical Workshop on ProtonAntiproton Collider Physics, 1989, p. 443.

5. W. C. Carithers, et al., "The CDF SVX: A Silicon Vertex Detector for a Hadron Collider", Nucl. Instrum. Methods A289, p. 388 (1990).

6. J. Skarha, et al, "Construction of the CDF Silicon Vertex Detector", in Conference Record of the 1991 IEEE Nucl. Sci. Symp., 1991, p. 426.

7. W. C. Carithers, et al, "The CDF Silicon Vertex Detector: An Overview and Test
Results", in Conference Record of the 1991 IEEE Nucl. Sci. Symp., 1991, p. 475.

8. B. Barnett, et al., "Progress in the Construction of the CDF Silicon Vertex Detector", Nucl. Instrum. Methods A315, p. 125 (1992).

9. F. Bedeschi, et al., "SVX Test Beam Results", Nucl. Instrum. Methods A315, p. 188 (1992).

10. S. A. Kleinfelder, et al., "A Flexible 128 Channel Silicon Strip Detector Instrumentation Integrated Circuit with Sparse Data Readout", IEEE Trans. on Nucl. Sci. NS-35, p. 171 (1988).

11. C. Haber, et al., "Design and prototyping of the Front End Readout System for the CDF Silicon Vertex Detector", IEEE Trans. on Nucl. Sci. NS-37, p. 1120 (1990).

12. S. M. Tkaczyk, et al., "Commissioning of the Control and Data Acquisition Electronics for the CDF Silicon Vertex Detector", in Conference Record of the 1991 IEEE Nucl. Sci. Symp., 1991, p. 753.

13. K. J. Turner, et al., "Control and Data Acquisition Electronics for the CDF Silicon Vertex Detector", in Conference Record of the 1991 IEEE Nucl. Sci. Symp., 1991, p. 888 . 